\title{
MIMO Capacity of an OFDM-based system under Ricean fading
}

\author{
Laxminarayana S. Pillutla and Sudharman K. Jayaweera
}

\begin{abstract}
In this paper we present the multiple-input multiple-output (MIMO) capacity results of an orthogonal frequency division multiplexing (OFDM) based system in correlated Ricean fading. Assuming that only receiver has the channel state information (CSI), the dependence of capacity on the propagation and system parameters are investigated. Our simulation and analytical results show that in general there would be a loss in ergodic capacity because of the Line-of-Sight (LOS) component, while high cluster angle spread increases the ergodic capacity.
\end{abstract}

\section{INTRODUCTION}

An OFDM-based MIMO system has the potential to drastically increase the capacity of wireless links with no additional power or bandwidth consumption. This is because an OFDM system has the ability to turn a frequency-selective MIMO fading channel into a set of parallel frequency-flat MIMO fading channels. This makes multichannel equalization remarkably simple. Also, by using a cyclic prefix (CP) (of duration greater than the multi-path delay spread of the channel) the intersymbol interference (ISI) can be completely eliminated. Using a broad-band fading channel model reported in [1], ergodic capacity of an OFDM based spatial multiplexing system under Rayleigh fading was derived assuming that the CSI is available only at the receiver in [2]. These expressions were then used to study the influence of propagation parameters (delay spread, cluster angle spread and total angle spread) and system parameters (number of antennas and antenna spacing) on capacity.

In this paper we consider the capacity of a similar OFDMbased system under a more general Ricean fading model. We obtain closed form expressions for the asymptotic capacity when only one LOS component is present. The dependence of asymptotic capacity on eigenvalue spread of the total correlation matrix (i.e. sum of correlation matrices of all the paths) was investigated in [2]. Here, we investigate how the eigenvalue spread, and hence the asymptotic capacity, would be affected by the Rice factor $\kappa$. Our analytical work followed by the simulation results reveal that in general the presence of a fading specular component reduces the ergodic capacity. This loss in ergodic capacity can be attributed to the reduction in the effective signal-to-noise ratio (SNR) due to the presence of LOS component.

The rest of the paper is organized as follows. Section II presents system model and assumptions. Section III presents the capacity of the given system under Ricean fading. Sections IV and V present the simulation results and conclusions.

\section{SYSTEM MODEL DESCRIPTION}

In general, using the discrete-time MIMO fading channel model we can write [2],

$$
\mathbf{y}[n]=\sum_{l=0}^{L-1} \mathbf{H}_{l} \mathbf{x}[n-l]
$$

where $n$ denotes the discrete-time index, $\mathbf{x}[n]$ denotes the discrete-time $N_{T} \times 1$ transmitted signal vector, $\mathbf{y}[n]$ denotes the discrete-time $N_{R} \times 1$ received signal vector and $\mathbf{H}_{l}$ represents the $l$-th tap of the channel impulse response.

\section{A. Propagation Scenario}

We assume that the receiver is at a height and is surrounded by a scatter-free environment resulting in spatially correlated fading at the receiver. On the other hand, it is assumed that 
the transmitter is surrounded by a number of scatterers and hence fading at the transmitter is spatially uncorrelated.

It is also assumed that the elements of the individual $\mathbf{H}_{l}$ are correlated but different scatterer clusters are uncorrelated, i.e.,

$$
\mathbf{E}\left[\operatorname{vec}\left\{\mathbf{H}_{l}\right\} \operatorname{vec}^{H}\left\{\mathbf{H}_{l^{\prime}}\right\}\right]=\mathbf{0}_{N_{R} N_{T}} \text { for } l \neq l^{\prime}
$$

where vec $\left\{\mathbf{H}_{l}\right\}=\left[\begin{array}{llll}\mathbf{h}_{l, 0}^{T} & \mathbf{h}_{l, 1}^{T} & \cdots & \mathbf{h}_{l, N_{T}-1}^{T}\end{array}\right]$, with $\mathbf{h}_{l, k}=$ $\left[h_{l, k}^{0} h_{l, k}^{1} \cdots h_{l, k}^{N_{R}-1}\right]^{T}$, denoting the $k$-th column of the matrix $\mathbf{H}_{l}$ and $\mathbf{0}_{N_{R} N_{T}}$ denoting the all zero matrix of size $N_{R} N_{T} \times N_{R} N_{T}$. Also, let us assume that each cluster has a mean angle of arrival $\bar{\theta}_{l}$, a cluster angle spread $\delta_{l}$ and a path gain of $2 \sigma_{l}^{2}$. The path gains are obtained from the power delay profile of the channel.

\section{B. Fading Statistics}

In a semi-urban environment it is realistic in assuming that there is one LOS component along with $L-1$ scattered signal components [1]. Thus, in our case we assume that $\mathbf{h}_{0, k}$ is distributed as $\mathcal{N}_{c}\left(\frac{\mu_{0}}{\sqrt{2}}(1+j), 2 \sigma_{0}^{2}\right)$ where $\mathcal{N}_{c}$ means a circularly symmetric complex Gaussian distribution. The other paths (i.e. for $l=1,2 \cdots L-1$ ) are assumed to be distributed as $\mathcal{N}_{c}\left(0,2 \sigma_{l}^{2}\right)$. Based on the above assumptions, $\mathbf{h}_{0, k}$ is said to have a Ricean distribution while the other paths are all assumed to be Rayleigh distributed. As in [2], we assume that the fading correlations are the same for all transmit antennas. Thus $\mathbf{R}_{l}=\mathbf{E}\left\{\mathbf{h}_{l, k} \mathbf{h}_{l, k}^{H}\right\}$. The entries of correlation matrix $\mathbf{R}_{l}$ can be written as

$$
\left[\mathbf{R}_{l}\right]_{m, n}=2 \sigma_{l}^{2} \rho_{l}\left((n-m) \Delta, \bar{\theta}_{l}, \delta_{l}\right)
$$

where we define $\rho_{l}\left(s \Delta, \bar{\theta}_{l}, \delta_{l}\right)=\mathbf{E}\left\{h_{l, k}^{r}\left(h_{l, k}^{r+s}\right)^{*}\right\}$ to be the fading correlation between two receiver antenna elements [I] Note that, $\rho_{l}$ is a function of the antenna spacing $\Delta$, mean angle of arrival $\bar{\theta}_{l}$ and the angular spread $\delta_{l}$. In general, it can be said that the spatial correlation increases with decreasing angular spread. Assuming a Gaussian distribution for angle of arrival, it has been shown in [1] that for a small angular spread the correlation can be approximated as

$$
\rho_{l}\left(s \Delta, \bar{\theta}_{l}, \delta_{l}\right) \approx e^{-j 2 \pi s \Delta \cos \left(\bar{\theta}_{l}\right)} e^{-0.5\left(2 \pi s \Delta \sin \left(\bar{\theta}_{l}\right) \sigma_{\theta, i}\right)^{2}} .
$$

The tap $\mathbf{H}_{0}$ of the channel, which is Ricean distributed can be written as

$$
\mathbf{H}_{0}=\mathbf{R}_{0}^{1 / 2} \mathbf{H}_{w, 0}+\overline{\mathbf{H}}_{0}
$$

where $\mathbf{R}_{0}$ is the covariance matrix of $\mathbf{H}_{0}, \mathbf{H}_{w, 0}$ is an uncorrelated $N_{R} \times N_{T}$ matrix with i.i.d complex normal entries of zero mean and unit variance and $\overline{\mathbf{H}}_{0}$ is an $N_{R} \times N_{T}$ matrix defined as for $m=1,2, \cdots, N_{R}$ and $n=1,2, \cdots, N_{T}$,

$$
\left[\mathbf{H}_{0}\right]_{m, n}=\frac{\mu_{0}}{\sqrt{2}}(1+j)
$$

The Rayleigh distributed $l$-th tap for $l=1,2, \cdots L-1$ can be written as

$$
\mathbf{H}_{l}=\mathbf{R}_{l}^{1 / 2} \mathbf{H}_{w, l} \text { for } l=1,2 \cdots L-1
$$

where $\mathbf{H}_{w, l}$ consists of complex normal entries of zero mean and unit variance. Also, for our further analysis we define the Rice factor $\kappa$ as $\kappa=\frac{\mu_{0}^{2}}{2 \sigma_{0}^{2}}$ and introduce the normalization $\mu_{0}^{2}+2 \sigma_{0}^{2}=1$. Thus, with this normalization we can write the mean and variance of the $\operatorname{LOS}$ component in terms of $\kappa$ as $\mu_{0}^{2}=\frac{\kappa}{\kappa+1}$ and $2 \sigma_{0}^{2}=\frac{1}{\kappa+1}$

\section{MUTUAL INFORMATION OF MIMO-OFDM BASED SYSTEM}

In this section we derive the mutual information of the MIMO-OFDM based system using the channel model described in the previous section. Throughout our analysis we assume that the length of the cyclic prefix is greater than the mutli-path time delay spread. Also the loss in spectral efficiency due to employing the cyclic prefix is neglected.

As in [2], we arrange the transmitted data symbols into frequency vectors $\mathbf{c}_{k}=\left[c_{k}^{(0)} c_{k}^{(1)} \cdots c_{k}^{N_{T}-1}\right]^{T}$ where $c_{k}^{(i)}$ denotes the data symbol transmitted from the $\mathrm{i}$-th antenna on the $\mathrm{k}$-th tone $(k=0,1, \cdots N-1)$. For our further analysis we define the following matrix $\mathbf{H}\left(e^{j 2 \pi \theta}\right)=\sum_{l=0}^{L-1} \mathbf{H}_{l} e^{-j 2 \pi l \theta}$ 
$(0 \leq \theta<1)$. Using these definitions the received code vector on the $k$-th tone can be written as

$\hat{\mathbf{c}}_{k}=\mathbf{H}\left(e^{j 2 \pi(k / N)}\right) \mathbf{c}_{k}+\mathbf{n}_{k}$, for $k=0,1, \cdots, N-1$

where $\mathbf{n}_{k}$ is the additive white Gaussian noise which is spatially and temporally white satisfying

$$
\mathbf{E}\left\{\mathbf{n}_{k} \mathbf{n}_{l}^{H}\right\}=\sigma_{n}^{2} \mathbf{I}_{N_{R}}
$$

where' $\mathbf{I}_{N_{R}}$ is the identity matrix of size $N_{R}$. With these definitions it can be shown as in [2] that the mutual information of an OFDM-based system with CSI available at the receiver is

$$
\begin{aligned}
I= & \frac{1}{N} \sum_{k=0}^{N-1} I_{k} \\
= & \frac{1}{N} \sum_{k=0}^{N-1} \log \left[\operatorname { d e t } \left(\mathbf{I}_{N_{R}}+\rho \mathbf{H}\left(e^{j 2 \pi(k / N)}\right)\right.\right. \\
& \left.\left.\mathbf{H}^{H}\left(e^{j 2 \pi(k / N)}\right)\right)\right]
\end{aligned}
$$

where $\rho=P /\left(N_{T} N \sigma_{n}^{2}\right)$ and $\mathrm{P}$ is the total transmitter power.

Note that we can write,

$$
\begin{aligned}
\mathbf{H}\left(e^{j 2 \pi(k / N)}\right) & =\mathbf{R}_{0}^{1 / 2} \mathbf{H}_{w, 0}+\overline{\mathbf{H}}_{0} \\
& +\sum_{l=1}^{L-1} \mathbf{R}_{l}^{1 / 2} \mathbf{H}_{w, l} e^{-j 2 \pi(k l / N)} .
\end{aligned}
$$

By denoting the first column of $\mathbf{H}\left(e^{j 2 \pi(k / N)}\right)$ as $\mathbf{h}\left(e^{j 2 \pi(k / N)}\right)$ it is straightforward to show as in [2] that

$$
\mathbf{E}\left\{\mathbf{h}\left(e^{j 2 \pi(k / N)}\right) \mathbf{h}^{H}\left(e^{j 2 \pi(k / N)}\right)\right\}=\mathbf{R},
$$

where $\mathbf{R}=\mathbf{R}_{0}+\mu_{0}^{2} \boldsymbol{\Psi}+\sum_{l=1}^{L-1} \mathbf{R}_{l}$ and $\boldsymbol{\Psi}$ is an $N_{R} \times N_{R}$ matrix of all ones. Thus, we note that the correlation matrix $\mathbf{R}$ is independent of $k$. Thus, we have shown that

$$
\mathbf{H}\left(e^{j 2 \pi(k / N)}\right)=\widetilde{\mathbf{R}}^{1 / 2} \mathbf{H}_{w}+\overline{\mathbf{H}}_{0}
$$

where $\mathbf{H}_{w}$ is $N_{R} \times N_{T}$ is uncorrelated complex Gaussian matrix with zero mean and unit variance and $\widetilde{\mathbf{R}}=\mathbf{R}-\mu_{0}^{2} \Psi$.

Hence, it follows that

$$
\begin{array}{r}
I_{k} \sim \log \left[\operatorname { d e t } \left(\mathbf{I}_{N_{R}}+\rho\left(\widetilde{\mathbf{R}}^{1 / 2} \mathbf{H}_{w}+\overline{\mathbf{H}}_{0}\right)\right.\right. \\
\left.\left.\left(\widetilde{\mathbf{R}}^{1 / 2} \mathbf{H}_{w}+\overline{\mathbf{H}}_{0}\right)^{H}\right)\right] .
\end{array}
$$

\section{A. Influence of LOS component on Capacity}

The ergodic capacity $C$ is defined as

$$
C=\mathbf{E}\left\{\frac{1}{N} \sum_{k=0}^{N-1} I_{k}\right\} .
$$

Since the distribution of $I_{k}$ is independent of k, from (14), $C$ can be further simplified as $C=\mathbf{E}\left\{I_{k}\right\}$ where $I_{k}$ is defined in (13).

Next, we resort to simple asymptotic analysis assuming that $N_{T}$ is large. In the limit of large transmit antennas it can be shown that

$$
C=\log \left[\operatorname{det}\left(\mathbf{I}_{N_{R}}+\bar{\rho} \tilde{\mathbf{R}}\right)\right]
$$

where $\bar{\rho}=P /\left(N \sigma_{n}^{2}\right)$.

To study the effect of $\kappa$ on the ergodic capacity let us consider the case of only one path. In this case, $\widetilde{\mathbf{R}}=\mathbf{R}_{0}$ where $\mathbf{R}_{0}$ corresponds to the covariance matrix of the 0 -th path. In the low SNR regime, the asymptotic capacity in (15) can be approximated as

$$
C \approx \log \left(1+\bar{\rho} \operatorname{Tr}\left(\mathbf{R}_{0}\right)\right)=\log \left(1+\frac{\bar{\rho}}{\kappa+1}\right)
$$

Thus, from (16) when the channel energy is fixed the presence of LOS component can decrease the capacity. This fact is further substantiated by our simulation results.

In the high-SNR case, we obtain

$$
C \approx \sum_{i=0}^{N R-1} \log \left(1+\bar{\rho} \lambda_{i}(\widetilde{\mathbf{R}})\right)
$$

The eigenvalue spread of $\tilde{\mathbf{R}}$ critically determines the ergodic capacity. In [2] it has been proved that the capacity is maximized when $\lambda_{i}(\widetilde{\mathbf{R}})=1 /\left(N_{R}\right)$ and any deviation from this constant value would result in a loss in ergodic capacity.

We next show as how the propagation parameters along with $\kappa$ influence the eigenvalues of $\widetilde{\mathbf{R}}$ and hence the ergodic capacity. It is straightforward to see that $\widetilde{\mathbf{R}}$ is a Toeplitz matrix, thus we can invoke Szegö's theorem [3] [4]. Using (4) we can write the limiting $\left(N_{R} \rightarrow \infty\right)$ distribution of the eigenvalues 

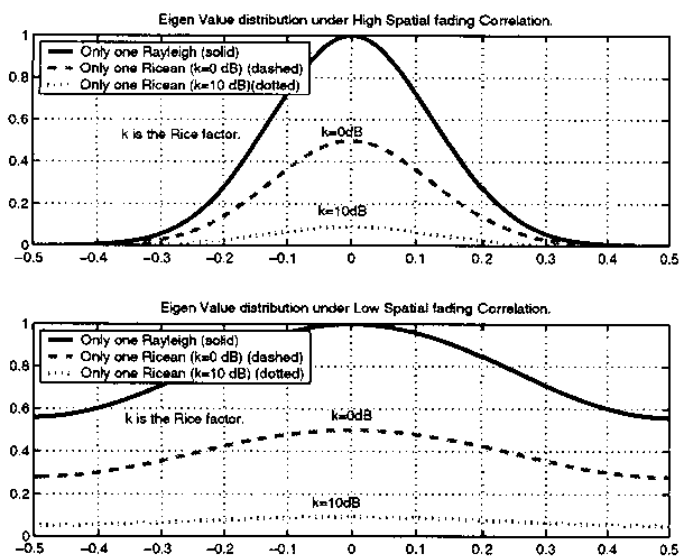

Fig. 1. Asymptotic Eigenvalue distribution with high and low spatial fading correlation

of $\widetilde{\mathbf{R}}$ as

$$
\begin{aligned}
\lambda(\nu)= & \frac{1}{\kappa+1} \vartheta_{3}\left(\pi\left(\nu-\Delta \cos \left(\theta_{0}\right)\right), e^{-(1 / 2)\left(2 \pi \Delta \sin \left(\theta_{0}\right) \sigma_{\theta, 0}\right)^{2}}\right) \\
& +\sum_{l=1}^{L-1} \vartheta_{3}\left(\pi\left(\nu-\Delta \cos \left(\theta_{l}\right)\right), e^{-(1 / 2)\left(2 \pi \Delta \sin \left(\theta_{l}\right) \sigma_{\theta, l}\right)^{2}}\right)
\end{aligned}
$$

where the third-order theta function is defined as $\vartheta_{3}(x, y)=$ $\sum_{n=-\infty}^{\infty} y^{n^{2}} e^{2 j n x}$ [5]. Although the expression for $\lambda(\nu)$ yields the eigenvalue distribution in the limiting case, in case of finite $N_{R}$, good approximations can be obtained by sampling $\lambda(\nu)$ uniformly on the unit circle [3]. We are now ready to study the impact of propagation parameters and $\kappa$ on the eigenvalue distribution and hence the ergodic capacity.

\section{B. Impact of cluster angle spread and Rice factor}

As stated before, in general the spatial fading correlation depends on the cluster angle spread $\left(\delta_{l}\right)$ and antenna spacing. In fact for $\sigma_{\theta, l}=0$ the correlation matrix collapses to a rank-1 matrix. For the sake of simplicity, first let us consider only one path. Fig.(1) shows the eigenvalue distribution under both high and low spatial fading correlation. As it is evident from this figure in the absence of cluster angle spread (i.e. high spatial fading correlation) the eigenvalue distribution is peaky and thus could result in a loss in ergodic capacity. Also, the presence of LOS component does not alter the eigenvalue distribution, but merely scales down the eigenvalue distribution by a factor of $\kappa+1$. This down scaling results in a reduction in the effective SNR by a factor of $\kappa+1$. But, in the presence of non-zero cluster angle spread (i.e. low spatial fading correlation) the eigenvalue distribution relatively flattens out and thus an improvement in ergodic capacity can be obtained. Thus, from this we conclude that in general the presence of a LOS component may reduce the capacity of an OFDM-based MIMO system in correlated fading.

\section{Simulation Results}

In every simulation example 1000 independent Monte Carlo runs were performed. We considered an OFDM based system in which the number of tones $(N)$ is equal to 512, the CP length was 64 . We also assume that the tap spacing in the simulation to be uniform and thus we model the power delay profile of the channel to be exponential. Also, for all the simulations we assume that only one tap is Ricean and the remaining taps are Rayleigh. The power of the LOS component was assumed to be $10 \mathrm{~dB}$ for all the cases. Finally, the SNR is defined as $\bar{\rho}=P /\left(N \sigma_{n}^{2}\right)$.

\section{A. Simulation Example 1}

The number of antennas $N_{T}=N_{R}=4$. To make the comparison fair we keep the total energy in the channel constant. In this example, we assume that the cluster angle spread $\sigma_{\theta, l}=0(l=0,1, \cdots, L-1)$. In the flat-fading case we set the mean angle of arrival $\bar{\theta}_{0}=\pi / 2$. In the multi-path case we assume a total angle spread of 90 degrees. As Fig. 2 indicates that in general the presence of a LOS component would reduce the capacity. For $L=6$ the capacity with pure Rayleigh fading channels and 1 Ricean +5 Rayleigh tap channel is almost the same. This is because when $L$ is large, the fraction of channel energy in the first path is very small. This makes the loss in effective SNR due to the LOS path component in this tap negligible. In general, the capacity of the system reduces with the increasing strength of LOS component as it would bring down the effective SNR. 


\section{B. Simulation Example 2}

Here, we investigated the effect of LOS component along with that of cluster angle spread (see Fig. 3). The parameters are same as in example 1 except that we assume a cluster angle spread of $\sigma_{\theta, l}=0.25(l=0,1, \cdots, L-1)$. As Fig. 3 shows, in general the cluster angle spread could result in an improvement in ergodic capacity. An interesting point to note is that the effect of cluster angle spread on the capacity is small in $L=1$ Ricean channel compared to that of Rayleigh channel. Beyond $L=3$, both Rayleigh and Ricean channels have almost the same capacity.

\section{CONCLUSIONS}

We considered the capacity of an OFDM-based MIMO system under correlated Ricean fading. Assuming that the CSI is available only at the receiver we derived expressions for the asymptotic capacity of such a system under the assumption that there is only one LOS component. We then derived the asymptotic eigenvalue distribution using which we analyzed the impact of LOS component along with other propagation parameters on the ergodic capacity.

Our analytical results suggest that in general the presence of LOS component could be detrimental to the ergodic capacity of the system. This fact was also illustrated using simulation results.

\section{ACKNOWLEDGEMENT}

This research was supported in part by Kansas NASA EPSCOR program under grant KUCR \# FED33144/KAN33145.

\section{REFERENCES}

[1] D. Asztély "On antenna arrays in mobile communication systems: Fast fading and GSM base station receiver algorithms," Royal Institute of Technology, Stockholm, Sweden, IR-S3-SB-96I1, 1996

[2] H. Bölcskei, D. Gesbert and A.J. Paulraj "On the capacity of OFDMbased spatial multiplexing systems," IEEE Trans. Commun., vol. 50, pp. 225-234, Feb. 2002.

[3] U. Grenander, and G. Szegö "Toeplitz forms and Their Applications," New York, Chelsea, 1984.

[4] R. M. Gray "Toeplitz and circulant matrices." Stanford Univ. ISL, 2000.

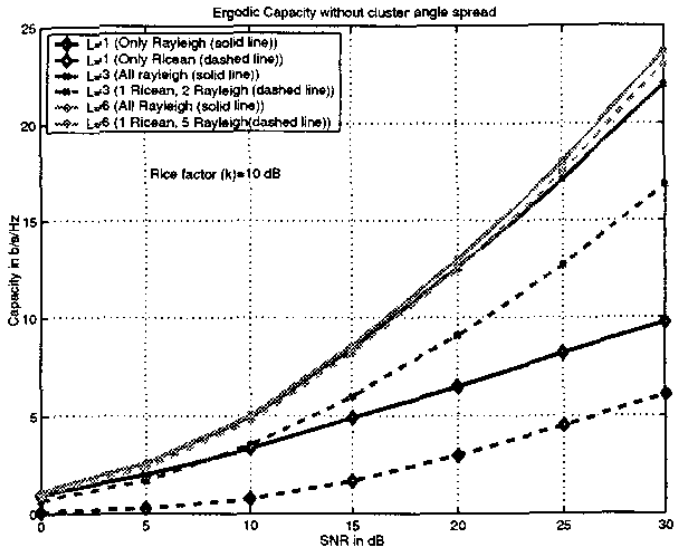

Fig. 2. Ergodic Capacity with small cluster angle spread

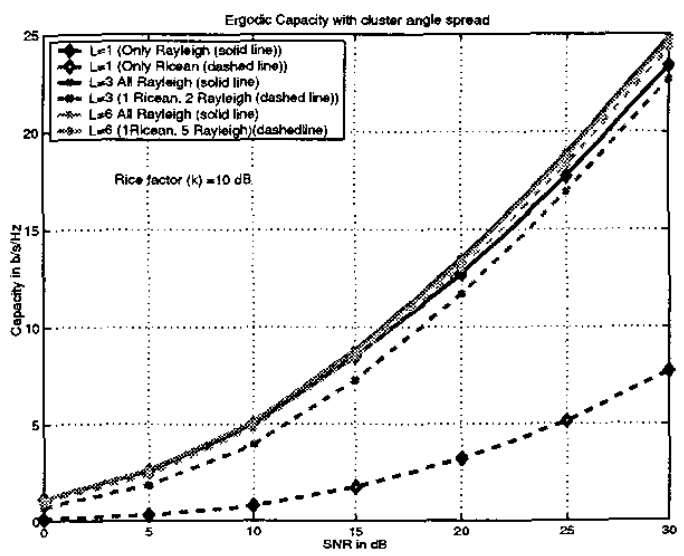

Fig. 3. Ergodic Capacity with large cluster angle spread

[5] I. S. Gradshteyn, and I. M. Ryzhik, "Table of Integrals, series, and products," Academic Press., New York, NY, USA, 1965. 